\title{
Miniature Mercury Contact Switches For Instrument Temperature Control
}

Thomas J. Bruno and Jerry G. Shepherd

National Bureau of Standards Boulder, CO 80303

\author{
In this short note, we describe the de- \\ sign and construction of several modifi- \\ cations of miniature mercury contact \\ switches for use in laboratory tempera- \\ ture control applications. Commercial \\ contact switches, or contact thermome- \\ ters as they are commonly called, are \\ limited in their application because of \\ their large size. The units which we \\ present here are much more compact
}

and are thus suitable for a wider range of applications. The limitations of the miniature contact switches in their present configurations are also discussed.

Key words: contact switch; contact thermometer; temperature controllers.

Accepted: September 17, 1985

\section{Introduction}

Temperature control for the components of lab. oratory instrumentation is achieved in a variety of ways. Depending on the level of accuracy required, contact switches, thermocouples, thermistors, and resistance devices are commonly used to control the power applied to heaters $[1,2]^{1}$. Thermistors and resistance devices are well suited to proportional control of the power input. Contact switches and thermocouples are easily applied to bipolar (on/off) control of heating elements.

All of these devices have their inherent limitations. For example, thermistors are usually limited to a maximum working temperature of $150^{\circ} \mathrm{C}$ $(423 \mathrm{~K})$. Thermo-couples are subject to aging ef-

About the Authors: Thomas J. Bruno and Jerry G. Shepherd are with the Thermophysics Division in the NBS National Engineering Laboratory. The work they describe was supported by the Gas Research Institute and the Department of Energy.

\footnotetext{
${ }^{1}$ Figures in brackets indicate literature references.
}

fects and become brittle and difficult to handle after operation at high temperature. The control circuitry associated with the use of thermistors and resistance devices is relatively expensive. Mercury contact switches (contact thermometers) have been used extensively for the control of stirred liquid bath temperature. The circuitry required for these simple on/off switches is inexpensive relay devices, which can control temperature with a precision approaching $\pm 0.005 \mathrm{~K}$ [3]. The large size and heat capacity of commercial mercury contact switches have limited their use in most other applications.

\section{Discussion}

In order to overcome some of these physical limitations and apply the contact switch to the 400 to $575 \mathrm{~K}$ region, we have designed and fabricated several miniature, high sensitivity fixed and adjustable mercury contact switches. These are shown in figure 1 . The units labeled $a$ and $b$ are fixed contact 


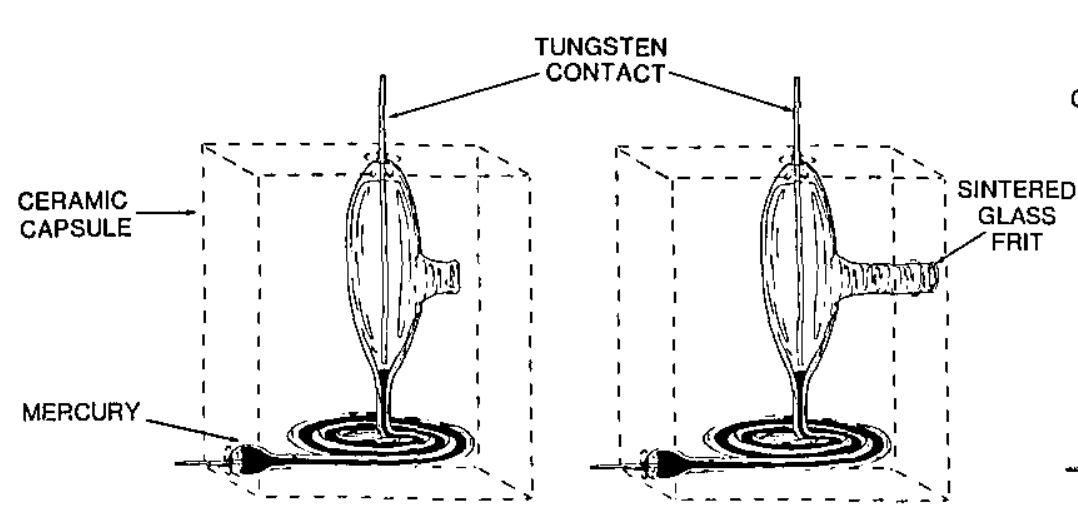

(1a) (1b)

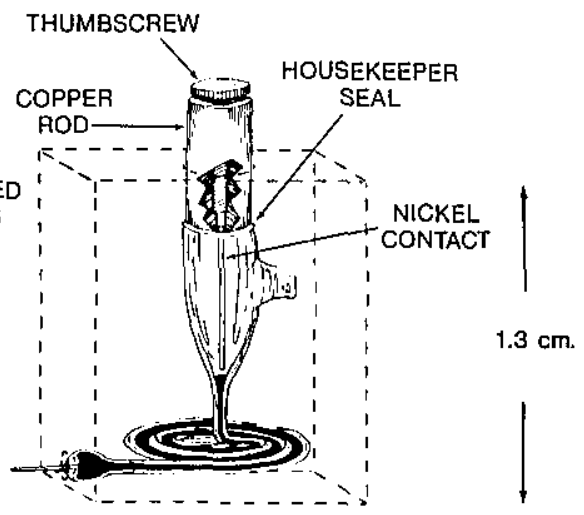

(1c)

Figure 1-The sealed, evacuated contact switch, a); ambient temperature modification of the switch, b); and the adjustable temperature switch, c).

switches which will regulate at a single specific temperature that must be set during fabrication. The body of each switch is made from borosilicate glass tubing ( $5 \mathrm{~cm}$ long, of $1.0 \mathrm{~mm}$ o.d. and $0.2 \mathrm{~mm}$ i.d.), and utilizes tungsten contacts.

The bulbs at either contact were made from uranium glass, in order to grade the seal to the tungsten. The switch shown in 1a is sealed under vacuum $\left(10^{-7} \mathrm{MPa}\right)$ and is suitable for use at elevated temperature. The upper bulb serves as an expansion reservoir, to prevent the unit from shattering in the unlikely event of a large temperature overshoot or a circuit failure. The unit shown in $1 \mathrm{~b}$ is a lower temperature modification of the fixed contact switch. The upper bulb is not evacuated, but is open to atmospheric pressure via a sintered glass frit.

With an adjustable contact switch as shown in 1c, the upper contact may be lowered or raised using the thumbscrew. The screw threads are covered with a commercial mixture of colloidal copper in vacuum grease, which lubricates the threads and insures good electrical contact. Since this switch is not sealed, its use is confined to lower temperature applications.

In practice, the contact switch is potted in a small pellet of a specially formulated aluminum-silicon oxide ceramic [4]. Alternatively, a high temperature silicone elastomer-aluminum mixture can be used. This pot provides good heat conduction and makes the switch more durable. This is desirable since the glass tubing is quite fragile.
The contact switch shown in figure 1a has been used to control the temperature of a chromatographic sampling system at $400 \mathrm{~K}[4,5]$. In this temperature region, the performance of thermistor based temperature controllers can be marginal. The contact switch is sensitive to changes in temperature on the order of $0.05 \mathrm{~K}$. The circuit (which responds to the contact switch) required to supply power to the heating elements is a simple solid state relay, shown in figure 2 [6].

The usefulness of these switches as substitutes for commercial mercury contact switches stems from the compactness of their design. They can be incorporated easily into instrument components which could not accommodate a larger commercial unit and where the use of a thermistor would provide marginal performance. The principal limitation of the switch in its present configuration is the inability to adjust the set point of the evacuated switch once the upper bulb is sealed. Thus, a separate switch is needed for each desired set point. This is not a serious problem for operations involving long term isothermal operation, but an adjustable sealed unit would be useful. Modifications along these lines are currently being pursued.

\section{References}

[1] Quin, T. J., Temperature, Academic Press, London (1983).

[2] Vassos, B. H., and G. W. Ewing, Analog and Digital Electronics for Scientists, New York: John Wiley and Sons (1980). 


\section{Journal of Research of the National Bureau of Standards}

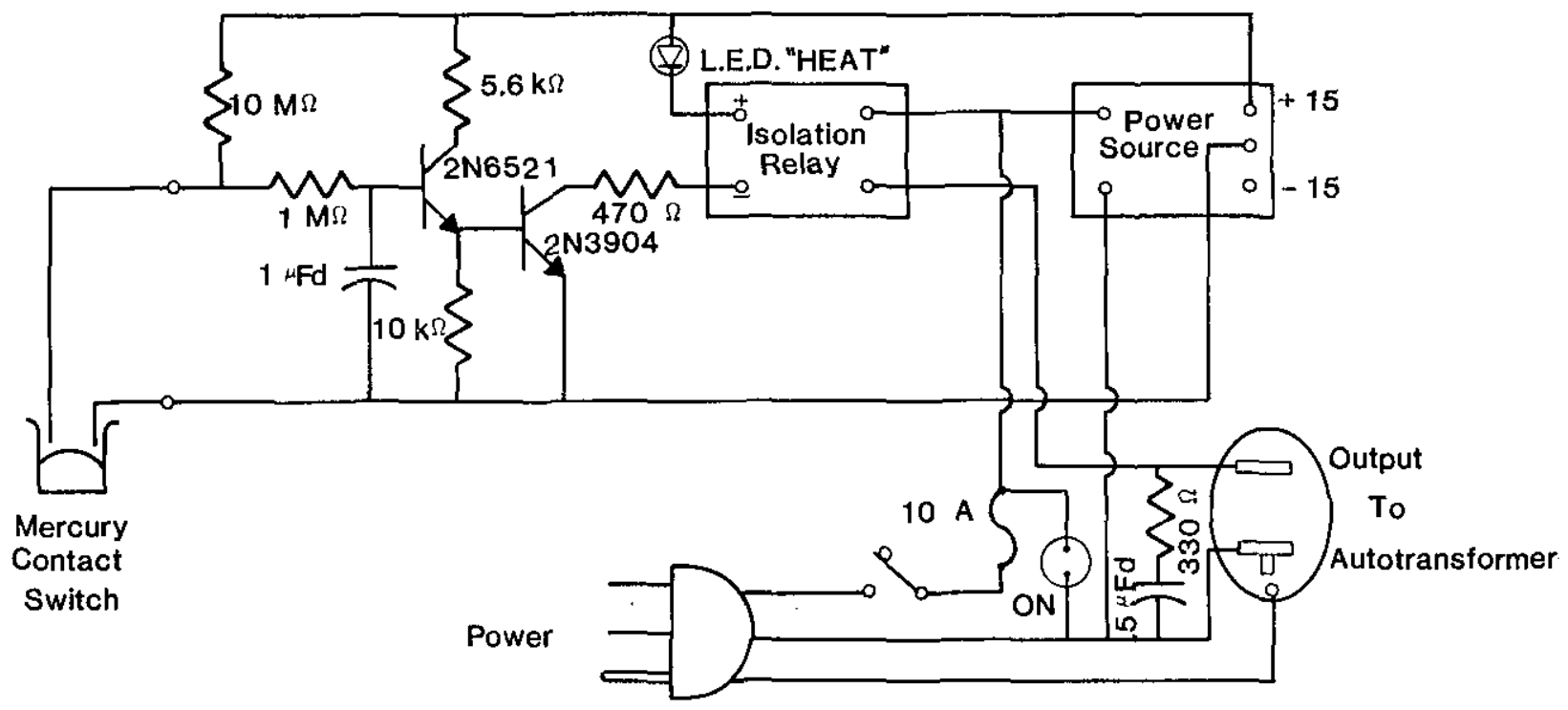

Figure 2-Circuit diagram for a simple solid state relay for use with the contact switches.

[3] Bruno, T. J.; D. E. Martire, M. W. P. Harbison, A. Nikolic, and C. F. Hammer, J. Phys. Chem. 87, 2425 (1983).

[4] Bruno, T. J., J. Chromatogr. Sci. 23, 325 (1985).

[5] Bruno, T. J., J. Res, Natl. Bur. Stand. (U.S.) 90, 127 (1985).

[6] Frederick, N. V., and J. McFarlane, Private Communication (1985). 